\title{
Spectropolarimetry and photometry of the early afterglow of the gamma-ray burst GRB 191221B *
}

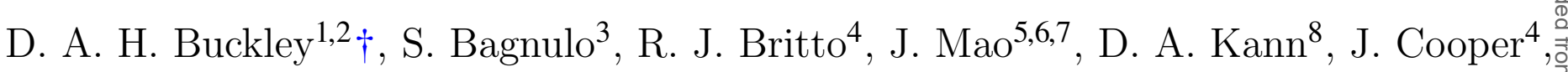
V. Lipunov ${ }^{9,10}$, D. M. Hewitt ${ }^{1,2}$, S. Razzaque ${ }^{11}$, N. P. M. Kuin ${ }^{12}$, I. M. Monageng ${ }^{1,2}$, S. Covino ${ }^{13}$, P. Jakobsson ${ }^{14}$, A. J. van der Horst ${ }^{15,16}$, K. Wiersema ${ }^{17,18}$, M. Böttcher ${ }^{19}$, S. Campana ${ }^{13}$, V. D’Elia ${ }^{20,21}$, E. S. Gorbovskoy ${ }^{9}$, I. Gorbunov ${ }^{9}$, D. N. Groenewald ${ }^{1,22}$, 岗 D. H. Hartmann ${ }^{23}$, V. G. Kornilov 9,10 , C. G. Mundell ${ }^{24}$, R. Podesta ${ }^{25,26}$, J. K. Thomas N. Tyurina 9 , D. Vlasenko9,10, B. van Soelen ${ }^{4}$ and D. $\mathrm{Xu}^{27}$

1 South African Astronomical Observatory, PO Box 9, Observatory Road, Observatory 7935, Cape Town, South Africa

2 Department of Astronomy, University of Cape Town, Private Bag X3, Rondebosch 7701, South Africa

3 Armagh Observatory $\& 3$ Planetarium, College Hill, Armagh, Northern Ireland, BT61 9DG, UK

${ }^{4}$ Department of Physics, University of the Free State, PO Box 339, Bloemfontein, 9300, South Africa

5 Yunnan Observatories, Chinese Academy of Sciences, 650011 Kunming, Yunnan Province, China

${ }^{6}$ Center for Astronomical Mega-Science, Chinese Academy of Sciences, 20A Datun Road, Chaoyang District, 100012 Beijing, China

${ }^{7}$ Key Laboratory for the Structure and Evolution of Celestial Objects, Chinese Academy of Sciences, 650011 Kunming, China

8 Instituto de Astrofísica de Andalucía (IAA-CSIC), Glorieta de la Astronomía s/n, 18008 Granada, Spain

9 M. V. Lomonosov Moscow State University, SAI, Physics Department, 13 Univeristetskij pr-t, Moscow 119991, Russia

10 M.V.Lomonosov Moscow State University, Physics Department, Leninskie gory, GSP-1, Moscow 119991, Russia

11 Centre for Astro-Particle Physics (CAPP) and Department of Physics, University of Johannesburg, PO Box 524, Auckland Park 2006, South Africa

12 Mullard Space Science Laboratory, Dept. of Space and Climate Sciences, University College London, Holmbury St Mary,

Dorking, RH5 6NT, UK

13 Brera Astronomical Observatory, via Bianchi 46, I-23807, Merate(LC), Italy

14 Centre for Astrophysics and Cosmology, Science Institute, University of Iceland, Dunhagi 5, 107, Reykjavk, Iceland

15 Department of Physics, The George Washington University, 725 21st St. NW, Washington, DC 20052, USA

16 Astronomy, Physics and Statistics Institute of Sciences (APSIS), The George Washington University, Washington, DC 20052, USA

17 Department of Physics, Gibbet Hill Road, University of Warwick, Coventry, CV4 7AL, UK

18 Department of Physics and Astronomy, University of Leicester, LE1 7RH, UK

19 Centre for Space Research, North West University, Potchefstroom, South Africa

${ }^{20}$ ASI-Space Science Data Center, via del Politecnico snc, 00133 Rome, Italy

${ }^{21}$ INAF - Osservatorio Astronomico di Roma, via Frascati 33, 00040 Monte Porzio Catone, Italy

22 Southern African Large Telescope Foundation, PO Box 9, Observatory Road, Observatory 7935, Cape Town, South Africa

23 Department of Physics and Astronomy Clemson University, Clemson, SC 29634-0978, USA

24 Department of Physics, University of Bath, Claverton Down, Bath BA2 7AY, UK

25 Observatorio Astronomico Felix Aguilar(OAFA), Avda Benavides s/n, Rivadavia, El Leonsito, Argentina

26 San Juan National University, Casilla de Correo 49, 5400 San Juan, Argentina

27 CAS Key Laboratory of Space Astronomy and Technology, National Astronomical Observatories, Chinese Academy of Sciences, Beijing 100101, China 


\begin{abstract}
We report on results of spectropolarimetry of the afterglow of the long gamma-ray burst GRB 191221B, obtained with SALT/RSS and VLT/FORS2, as well as photometry from two telescopes in the MASTER Global Robotic Network, at the MASTER-SAAO (South Africa) and MASTEROAFA (Argentina) stations. Prompt optical emission was detected by MASTER-SAAO $38 \mathrm{~s}$ after the alert, which dimmed from a magnitude (white-light) of $\sim 10$ to 16.2 mag over a period of $\sim 10 \mathrm{ks}$, followed by a plateau phase lasting $\sim 10 \mathrm{ks}$ and then a decline to 18 mag after $80 \mathrm{ks}$. The light curve shows complex structure, with four or five distinct breaks in the power-law decline rate. SALT/RSS linear spectropolarimetry of the afterglow began $\sim 2.9 \mathrm{~h}$ after the burst, during the early part of the plateau phase of the light curve. Absorption lines seen at $\sim 6010 \AA$ and $5490 \AA$ are identified with the Mg II $2799 \AA$ line from the host galaxy at $z=1.15$ and an intervening system located at $z=0.96$. The mean linear polarisation measured over $3400-8000 \AA$ was $\sim 1.5 \%$ and the mean equatorial position angle $(\theta) \sim 65^{\circ}$. VLT/FORS2 spectropolarimetry was obtained $\sim 10 \mathrm{~h}$ postburst, during a period of slow decline $(\alpha=-0.44)$, and the polarisation was measured to be $p=1.2 \%$ and $\theta=60^{\circ}$. Two observations with the MeerKAT radio telescope, taken 30 and 444 days after the GRB trigger, detected radio emission from the host galaxy only. We interpret the light curve and polarisation of this long GRB in terms of a slow-cooling forward-shock.
\end{abstract}

Key words: High energy astrophysics; Gamma-ray bursts; Magnetie fields; Polarimetry; Shocks; Jets

\section{INTRODUCTION}

Gamma-ray bursts (GRBs) are fast, high-energy transient phenomena which, during the sub-second to few hundred seconds duration of the event, are the most luminous sources of $\gamma$-rays in the Universe, with a typical energy release of $\sim 10^{51}$ ergs. GRBs are the result of the collapse of massive, highly evolved stars, or the merger of compact objects, with a significant number, particularly the so-called "long-soft" GRBs, linked to core-collapse supernovae (for a review, see Cano et al. 2017). Accretion onto a resulting compact object, like a black hole or neutron star, produces powerful ultra-relativistic jets which, through dissipation processes like shocks or magnetic reconnection, produce prompt $\gamma$-ray emission (for reviews of GRBs and GRB physics, see e.g. Gehrels et al. 2009, Gehrels \& Razzaque 2013, Gao et al. 2013, Wang et al. 2015, Kumar \& Zhang 2015).

The resulting rapidly expanding ejecta of a GRB, after the prompt emission phase, collides with the surrounding medium, producing long-lasting emission called an afterglow, detected across the whole electromagnetic spectrum (e.g., Piran 1999; Mészáros 2002; Piran 2004). At the onset of the collision-driven afterglow, shocks are formed, one forward-propagating into the external medium, while another, shorter-lived reverse shock propagates backward into the jet (Sari \& Piran 1999; Kobayashi 2000). The interaction between the ejecta and the surrounding medium may be quantified by several micro-physical parameters, such as the degree of the ejecta's magnetisation, $\sigma_{B}$. This is the ratio of magnetic to kinetic energy and in the matter-dominated regime model for a standard fireball, $\sigma_{B}<1$, and therefore shocks are plasma-dominated (Rees \& Meszaros 1994; Gomboc et al. 2008). With increasing $\sigma_{B}$ the magnetic energy becomes significant, and the reverse shock develops un-

* Based on observations made with the Southern African Large Telescope (SALT) and the MeerKAT radio telescope array.

$\dagger$ E-mail: dibnob@saao.ac.za til it reaches a maximum at $\sigma_{B} \sim 0.1$, whereupon it weakens and is suppressed for $\sigma_{B} \geq 1$ (Giannios et al. 2008 and references therein). For a highly magnetised outflow, the deceleration region has a $\sigma_{B} \gg 1$ and so the jet is Poynting-flux dominated.

The prompt emission has been suggested to result from magnetic energy dissipation, where the ejecta entrains ordered magnetic fields (Lyutikov et al. 2003 and references therein). This emission, and the early-time afterglow emission from reverse shocks, may show high levels of linear polarisation in some cases (e.g. Steele et al. 2009; Mundell et al. 2013; Troja et al. 2017). Optical polarisation calibration is well-established, with comparison of GRB measurements and field stars providing additional robustness to detections. More controversial are claims of prompt gamma-ray emission polarisation, with reported measurements spanning the full range from zero to $100 \%$ polarisation, and significant disagreement in the parameter distributions derived with different gamma-ray instruments (e.g., Kole et al. 2020). In a Poynting fluxâĂŞdominated magnetised jet outflow, the early-time emission is expected to be highly polarised. This is thought to be due to the presence of pre-existing magnetic fields, advected from the central source (e.g., see Zhang \& Kobayashi 2005 and references therein). For baryon-dominated jets, the magnetic fields generated locally in shocks are tangled, resulting in unpolarised emission for on-axis jets and low polarisation for edge-on jets (Medvedev \& Loeb 1999; Sari 1999; Mao \& Wang 2017). Early-time polarisation measurements of GRB afterglows are therefore crucial for probing the details of the shock physics and for discriminating between different jet models (e.g., Mundell et al. 2013). A review of past GRB prompt and afterglow polarisation measurements can be found in Covino \& Götz (2016).

At late times, in the forward shock regime of the afterglow, the predicted polarisation at optical wavelengths is a strong function of the viewing geometry of the jet (i.e. the opening angle of the jet and our viewing angle with respect 
to the jet center direction), the internal structure of the jet, and the order and strength of the magnetic field (both within the shock and normal to the shock). Most of these parameters influence the total flux light curve only mildly, but have a large effect on the polarisation as a function of time (see e.g. Rossi et al. 2004), leading to models for the polarisation (amplitude and angle) as a function of time, which can be tested with high quality data of individual afterglows, as well as the ensemble of measurements of a large number of sources (e.g. Wiersema et al. 2014; Gill \& Granot 2020; Stringer \& Lazzati 2020; Teboul \& Shaviv 2020). There are now a few dozen GRBs for which optical polarisation has been detected in their afterglows, and a relatively rich phenomenology is found. Generally speaking, most forwardshock-afterglow polarisation measurements show low levels of linear polarisation (at most a few percent), in many cases with clear signs of variability in both polarisation angle and amplitude. In some high signal-to-noise cases, evidence exists for polarimetric amplitude and angle variability associated with bumps in the optical and X-ray total flux light curve (e.g. Greiner et al. 2003; Wiersema et al. 2012). Some afterglows exhibit polarisation signatures supporting the model predictions for homogeneous jets with random fields (e.g. a 90 degree polarisation angle flip, Wiersema et al. 2014), whereas some GRBs more closely follow structured jet models instead (which show no such 90 degree angle change), with possibly an ordered magnetic field component normal to the shock (e.g. Gill \& Granot 2020; Teboul \& Shaviv 2020). In many cases it is not practically possible to obtain high quality polarimetry over a long time period, as most afterglows fade rapidly, and therefore single-epoch measurements of a large number of sources remain important to establish the overall parameter space. The interpretation of polarisation data relies on good multi-wavelength light curves (e.g. to measure the jet collimation angle and the position of the synchrotron break frequencies), and it is therefore important to increase the sample of afterglows with both polarimetric measurements and well-sampled light curves, such as the data set presented in this paper.

A relatively poorly explored polarimetric probe of afterglow physics is multi-wavelength polarimetry, combining near-simultaneous polarisation measurements spanning a wide range of wavelengths, which opens a new window on the afterglow physics (e.g. Toma et al, 2008). Recently, instruments at long wavelengths have become sufficiently sensitive to deliver on this promise for both reverse and forward shock regimes (e.g. Laskar et al. 2019; Urata et al. 2019; van der Horst et al. 2014). At optical wavelengths, spectro-polarimetry has some diagnostic power in this way as well, particularly if (by chance) any of the synchrotron break frequencies (e.g., the synchrotron cooling frequency) are present near the optical band. Spectropolarimetry also helps to quantify a key contaminant in afterglow polarimetry studies: the polarisation induced by dust in the GRB host galaxy and in our own Galaxy. Multi-colour polarimetry or spectro-polarimetry are the best ways to quantify this contribution, which is likely to play a non-negligible role in the retrieved polarisation distribution of afterglows and their physical interpretation (see, e.g. Lazzati et al. 2003; Covino \& Götz 2016; Wiersema et al. 2014; Kopač et al. 2015; Jordana-Mitjans et al. 2020). To date, the number of afterglows studied with optical spectropolarimetry is limited to just a few cases, e.g. GRB 020813 (Barth et al. 2003), GRB 021004 (Wang et al. 2003), GRB 030329 (Greiner et al. 2003) and GRB 080928 (Covino \& Götz 2016). In addition, some spectropolarimetric measurements have been performed for the SNe accompanying GRBs (e.g., GRB 060218, Maund et al. 2007).

Here we report on follow-up optical photometry, spectroscopy and spectro-polarimetry of the optical afterglow of GRB 191221B. Prompt $\gamma$-ray emission was detected on 2019-12-21 20:39:11.42 ( $\pm 0.01 \mathrm{~s})$ UT by AGILE (Longo et al. 2019) and on 2019-12-21 20:39:13 UT by Swift/BAT (Laha et al. 2019).

\section{GRB 191221B}

GRB 191221B was detected and first reported by the Neil Gehrels Swift Observatory (henceforth Swift) Burst Alert Telescope (BAT, Barthelmy et al. 2005) on 2019-12-21 at 20:39:13 UT (Laha et al. 2019). Swift slewed immediately to the burst, repointing its narrow-field instruments, the Xray telescope (XRT, Burrows et al. 2005) and the Ultraviolet and Optical Telescope (UVOT, Roming et al. 2005). A bright afterglow was detected both by XRT and UVOT. The BAT light curve shows a complex prompt emission structure with a duration $\mathrm{T}_{90}=48 \pm 16 \mathrm{~s}$ in the $15-350 \mathrm{keV}$ band, and the spectrum can be fit with a simple power-law with index $-1.24 \pm 0.05$

The fluence of GRB 191221B was in the top third of all BAT-detected bursts (Sakamoto \& Swift-BAT Team 2019). The prompt emission was also reported by AGILE/MCAL (Longo et al. 2019), AstroSat CZTI (Gaikwad et al. 2019), Insight-HXMT/HE (Xue et al. 2019), Konus-Wind (Frederiks et al. 2019) and CALET (Sugita et al. 2019). Although the AstroSat CZTI is, in principle, able to observe $\gamma$-ray polarisation, the orientation of the spacecraft was not favorable for a detection of GRB 191221B. The earliest prompt $\gamma$-ray detection was obtained by CALET, at 20:39:05 UTC, which we adopt as the time of the burst, $T_{0}$. This was followed by the first reported ground-based detection of a bright (unfiltered magnitude $=10.5 \mathrm{mag}$ ) optical transient by MASTER-SAAO at 20:41:35 UT, $150 \mathrm{~s}$ after the CALET burst detection (Lipunov et al. 2019d), although earlier data points were subsequently determined (see next section). The source was so bright that UVOT was able to acquire a grism spectrum, which led to a measurement of the redshift of $z=1.19$ (Kuin \& Swift/UVOT Team 2019), later confirmed and refined by the ESO/VLT X-shooter spectrograph to $z=1.148$ by Vielfaure et al. (2019), who also reported the presence of an intervening system at $z=0.961$. The afterglow was also detected in the radio band by ALMA (11.1 hrs after the trigger, Laskar \& a larger collaboration 2019), ATCA (17.5 hrs after the burst, Laskar 2019), and MeerKAT (30 days after the trigger, Monageng et al. 2020).

\section{MASTER PHOTOMETRY OF GRB 191221B}

The MASTER Global Robotic Telescope Network (Lipunov et al. 2010, 2019a) began to observe the GRB 


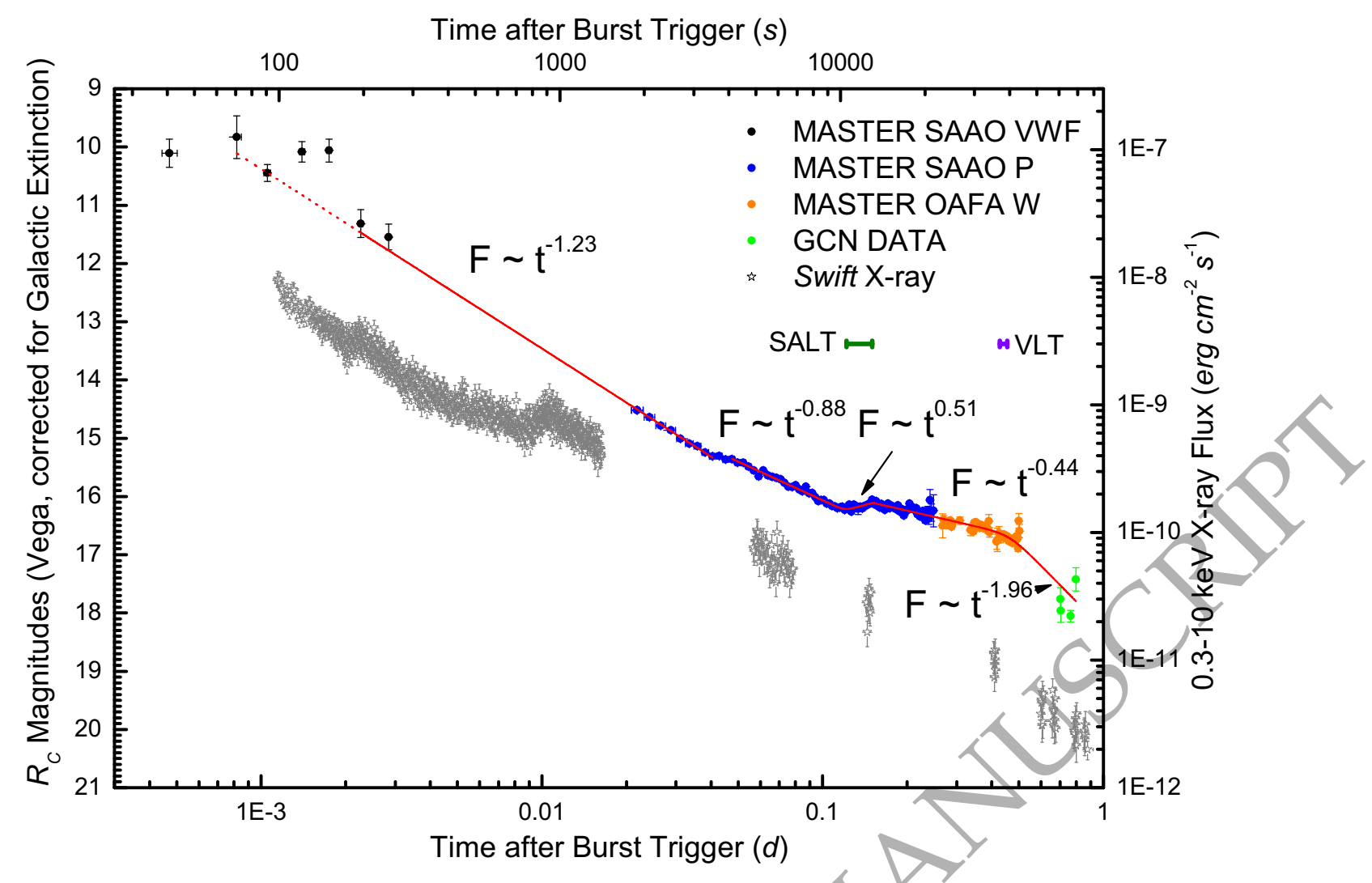

Figure 1. Light-curve evolution of GRB 191221B determined by the MASTER-SAAO and MASTER-OAFA facilities (as well as several other ground-based observations, labelled GCN, see text for references), as well as by Swift XRT in the $0.3-10 \mathrm{keV}$ range. Time is given in days as well as seconds after the $C A L E T$ burst trigger time, namely $T_{0}=20: 39: 05$ UT. The spectro-polarimetric coverage by SALT/RSS (10, $472-12,925$ s post-burst) and VLT/FORS2 (36, $906-39,307$ s post burst) is indicated by a green and a purple bar, respectively.

191221B error box at 2019-12-21 20:39:43 UT, 38 s postburst, using the very wide field cameras (VWFC) at MASTER-SAAO, in South Africa (Lipunov et al. 2019d). The VWFC enables wide-field coverage in white light (W) with constant sky imaging every $5 \mathrm{~s}$, which is crucial for GRB prompt detections (Gorbovskoy et al. 2010; Kornilov et al. 2012; Sadovnichy et al. 2018). The brightness of the optical afterglow at discovery was $W=10.3 \mathrm{mag}$ and it remained at this brightness for $\sim 150$ s post-burst, thereafter rapidly declining in brightness.

Observations at MASTER-SAAO, using one of the MASTER-II telescopes (a pair of $0.4 \mathrm{~m}$ twin telescopes), started at 2019-12-21 21:09:03 UT ( 1798 s postburst) using a polarizer and clear filter (Lipunov et al. 2019b), although observations were only possible with one of the pair of telescopes due to a CCD camera being non-operational. The position of the optical afterglow was determined by the MASTER auto-detection system (Lipunov et al. 2010, 2019a) from these observations, when GRB 191221B had dimmed to $W=14.4 \mathrm{mag}$. The coordinates of the optical counterpart were determined to be RA, Dec. (J2000) $=10^{h} 19^{m} 19.24^{s},-38^{\circ} 09^{\prime} 28.7^{\prime \prime}$ and the optical transient was given the name MASTER OT J101919.24-380928.7 (Lipunov et al. 2019c). MASTER-SAAO observations continued until 21,367 s ( $5.93 \mathrm{~h})$ post-burst, by which time GRB 191221B had faded to $W=16.45$ mag. Observations then began with the 0.4-m MASTER-OAFA telescope, in
Argentina, 23,017 s ( $6.39 \mathrm{~h})$ post-burst, following the completion of the MASTER-SAAO observations, and continued until $43,324 \mathrm{~s}(\sim 12 \mathrm{~h})$ post-burst, at which time the afterglow was at $W=16.77 \mathrm{mag}$.

The MASTER clear band magnitude, $W$, is best described by the Gaia $G$ filter. We performed two similar photometric calibration procedures using two different sets of reference stars from the Gaia DR2 catalogue, seven for the VWFC images and nine for the MASTER II telescope images. These were used to determine the measurement error (see Troja et al. 2017 for a more detailed photometric error determination description). After astrometric calibration of each image, we performed standard aperture photometry using ASTROPY/PHOTUTILS (Bradley et al. 2016).

In Figure 1 we show the optical light curve evolution of GRB 191221B determined by the MASTER-SAAO and MASTER-OAFA facilities and including subsequent brightness measurements reported in the GCN circulars, as well as by Swift XRT in the $0.3-10 \mathrm{keV}$ range, taken from the Burst Analyser (Evans et al. 2010) ${ }^{1}$. The light curve of GRB 191221B shows complex breaks in its decline rate, characterised by a general decrease in flux with time, following a sequence of power-laws, $F \propto t^{\alpha}$. The initial decline rate has $\alpha=-1.23 \pm 0.04$ (measured starting $\approx 1,900 \mathrm{~s}$ af-

\footnotetext{
1 https://www.swift.ac.uk/burst_analyser/00945521/
} 
Table 1. Power-law slopes of the optical afterglow light curve of GRB 191221B measured at different phases.

\begin{tabular}{rrr}
$\mathrm{T}_{\text {start }}(\mathrm{s})$ & $\mathrm{T}_{\text {end }}(\mathrm{s})$ & \multicolumn{1}{c}{$\alpha$} \\
\hline 1,890 & 3,500 & $-1.23 \pm 0.04$ \\
3,500 & 10,000 & $-0.88 \pm 0.02$ \\
10,000 & 12,000 & $0.51 \pm 0.14$ \\
12,000 & 40,000 & $-0.44 \pm 0.01$ \\
40,000 & 70,000 & $-1.96 \pm 0.14$ \\
\hline
\end{tabular}

ter the trigger, but a back-extrapolation shows this decay joins with the early very bright emission), which flattens to $\alpha=-0.88 \pm 0.02$ at $\sim 0.83 \mathrm{~h}$ post-burst. This is followed by a short lived re-brightening, lasting for $\sim 0.55 \mathrm{~h}$ and rising with $\alpha=0.51 \pm 0.14$. The afterglow of GRB 191221B then declined slowly, with $\alpha=-0.44 \pm 0.01$ until $\sim 11 \mathrm{~h}$ post-burst, thereafter breaking and declining more rapidly with $\alpha=-1.96 \pm 0.14$, where the latter was determined using magnitudes reported in the GCNs (Romanov 2019; Gendre 2019; Kong 2019). Note the exact value of the latter slope is not well-determined and may change with the addition of further data beyond one day. Details of the power-law slopes are presented in Table 1.

\section{SPECTROPOLARIMETRY}

\subsection{SALT/RSS}

Observations of the optical afterglow of GRB 191221B were obtained with the Southern African Large Telescope (SALT; Buckley et al. 2006) using the Robert Stobie Spectrograph (RSS, Burgh et al. 2003) in spectropolarimetry mode (Nordsieck et al. 2003). The observations were obtained between 23:34 and 00:15 UTC on 2019 December 21, starting 2 hours $54 \mathrm{~min}$ after the GRB alert. The observations were carried out during the re-brightening phase of the light curve.

Four consecutive exposures of $600 \mathrm{~s}$ were obtained at four different orientations of a $1 / 2$ waveplate retarder $\left(0^{\circ}\right.$, $22.5^{\circ}, 45^{\circ}$ and $67.5^{\circ}$ ) and the results were analysed to determine the Stokes Q and U parameters, the magnitude of the linear polarisation, $p$, and the position angle of the $\mathrm{E}$ vector, $\theta$. We used the PG300 transmission grating and a 1.' 5 wide slit, which gave a wavelength coverage of $3400-8000$ $\AA$ at a resolution of $\sim 16 \AA$. The spectrograph slit was oriented to a position angle of $45^{\circ}$ to allow the nearby $\left(\sim 1^{\prime}\right)$ bright $(B=14.7, R=13.2 \mathrm{mag})$ reference star, USNO A2 0 0450-11150896, to be measured simultaneously with GRB 191221B. This allows for subtraction of the interstellar polarisation component.

The spectropolarimetry reductions were carried out using an adaptation of the beta version of the polSALT ${ }^{2}$ software $^{3}$ and the results are shown in Figure 2 and Figure 3 at two resolutions ( $50 \AA$ and $100 \AA$ ) for the polarisation parameters, the latter figure including the measurements of

${ }^{2}$ https://github.com/saltastro/polsalt

3 We used POLSALT version 20171226 (including specpolextract_dev version 20180524), based on PYSALT v0.5dev a nearby field star. We found that GRB 191221B was polarised at an average level of $p=1.5 \%$, with a variation of $\pm 0.5 \%$, and $\theta=65^{\circ}$ with a variation of $\pm 10^{\circ}$, over the range $3900-8000 \AA$.

Foreground polarisation due to the ISM was estimated from the nearby $\left(\sim 50^{\prime \prime}\right.$ in the SE direction) reference star, USNO A2.0 0450-11150896 (Gaia DR2 5444869271098575232 ), which was also placed on the spectrograph slit. The mean polarisation was $p \sim 0.3 \%$ and $\theta \sim 130^{\circ}$ over the range $4300-7300 \AA$.

\subsection{VLT/FORS2}

Spectropolarimetry of GRB 191221B was also obtained using the FORS2 instrument attached at the Cassegrain focus of the Unit 1 (Antu) of the ESO Very Large Telescope. Observations began $\sim 10 \mathrm{~h}$ after the burst (from 06:54 UT on 2019 December 22), during the slow decline phase of the afterglow, where $\alpha=-0.44$. With the 300 grism (with no order separating filter) and a 1.' 5 slit width. FORS2 observations cover the spectral range from about 3200 - $9200 \AA$ with a spectral resolution of $\sim 17 \AA$. The observations were performed using the beam-swapping technique, and the total exposure time was $2400 \mathrm{~s}$, equally split in four exposures with the $\lambda / 2$ waveplate at position angles $0^{\circ}, 22.5^{\circ}$, $45^{\circ}$ and $67.5^{\circ}$. Observations were obtained with the E2V blue-optimised CCD mounted on the instrument. Because of the relatively/low spectral resolution, fringing at longer wavelengths did not strongly affect the spectrum. Data were reduced using IRAF routines, as described in Sect. 2.3 of Bagnulo et al. (2017).

The correct alignment of the polarimetric optics was obtained by observing the standard star for linear polarisation, Ve 6-23 (e.g. Fossati et al. 2007) on the same night. In the same slit as the main target, we also observed a foreground star, slightly fainter than the afterglow of GRB 191221B, which showed low polarisation (average $p=0.2 \%$ over 4000 $7200 \AA$ ). This reference star was different from the one observed by SALT, being only 5" from GRB 191221B and also considerably fainter. The polarisation values of both reference stars are the same, within the uncertainties, indicating a low level of ISM polarisation $(\leqslant 0.3 \%)$.

The results are shown in Figure 4, where the polarimetric measurements were determined after binning the data to $25 \AA$ per bin. The mean polarisation values determined for GRB $191221 \mathrm{~B}$ were $p=1.2 \%$ and $\theta=60^{\circ}$, slightly less than the SALT/RSS values obtained $\sim 7 \mathrm{~h}$ earlier.

\section{SPECTRAL LINES}

Significant absorption lines are seen in both the RSS and FORS2 spectra, the strongest located around $6010 \AA$ which was identified as the Mg II $2799 / 2802 \AA$ doublet by Vielfaure et al. (2019) based on VLT/X-Shooter observations. They concluded that this implied a host galaxy redshift of $z=1.148$. A weaker system of absorption lines, around $5490 \AA$, was also seen, corresponding to the same $\mathrm{Mg}$ II doublet at a lower redshift of $z=0.961$, from an intervening system along the line-of-sight.

The GRB 191221B spectra are shown in Figure 5, where proposed line identifications are also shown. Line fits 


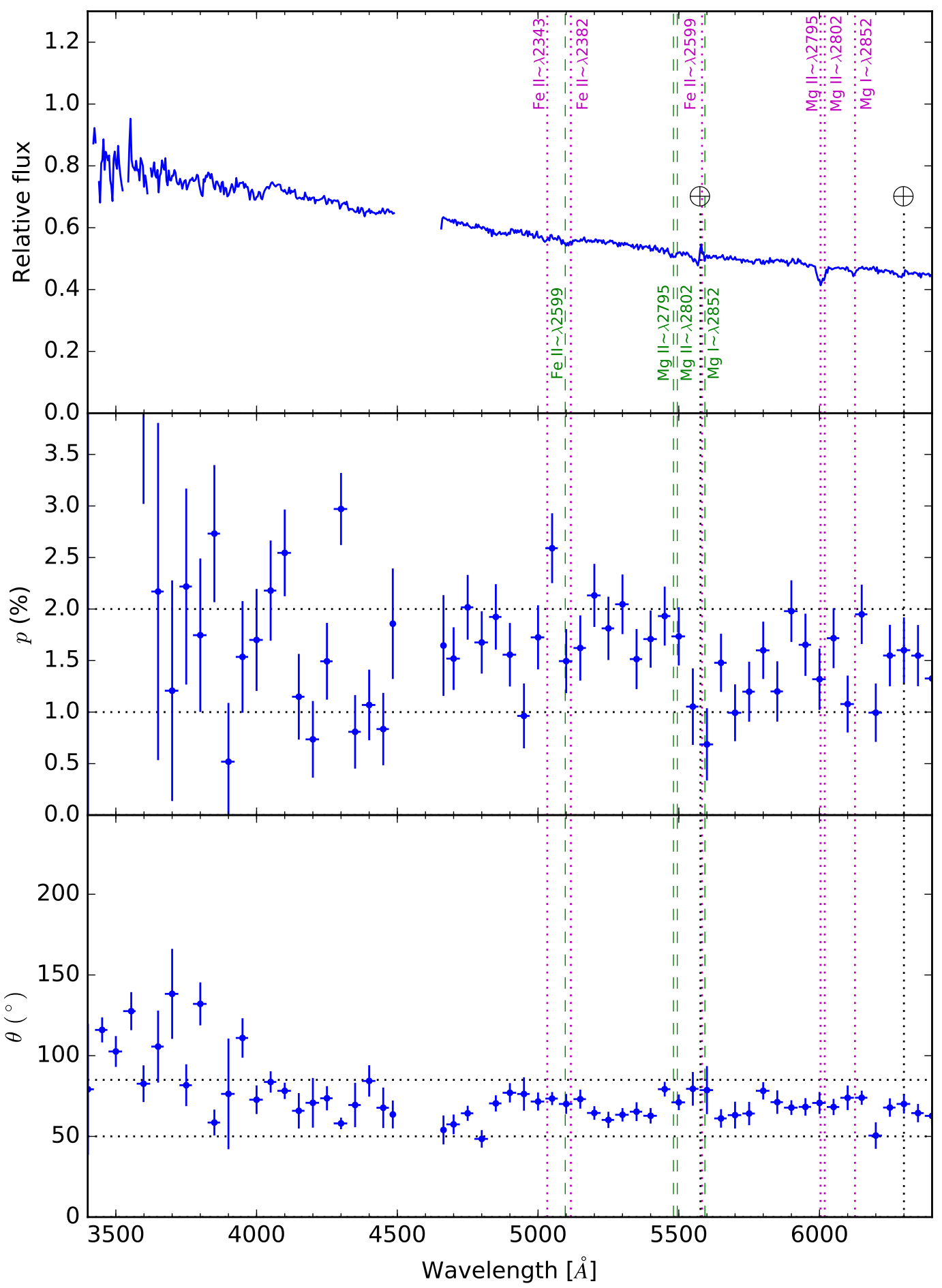

Figure 2. SALT/RSS spectropolarimetry of GRB 191221B covering 3400-6300 $\AA$, where $p \& \theta$ were determined after binning the data to $50 \AA$ (see text). Absorption features from the host galaxy $(z=1.15$; magenta dotted lines) and an intervening galaxy $(z=0.96$; green dashed lines) are indicated. Telluric lines are indicated with black dotted lines. There are no data from $\sim 4500-4650 \AA$ due to a chip gap in the CCD mosaic. 


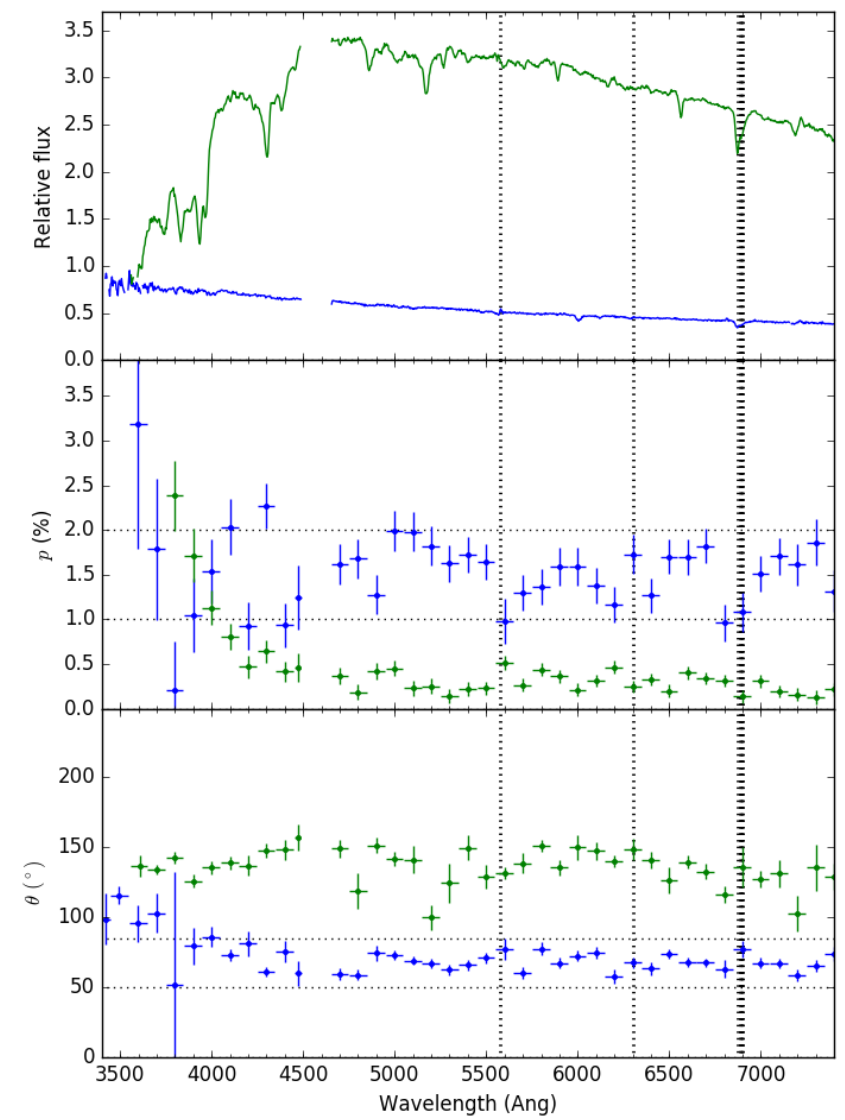

Figure 3. SALT/RSS spectropolarimetry of GRB 191221B (blue) and the nearby bright field star (green), where $p \& \theta$ were determined after binning the data to $100 \AA$ (see text). Telluric lines are indicated with thick black dotted lines. There are no data from $\sim 4500-4650 \AA$ due to a chip gap in the CCD mosaic.

were attempted on both spectra and the results are presented for the higher S/N data from FORS2 in Table 2. Three close pairs of lines resolved in the FORS2 spectra (Fe

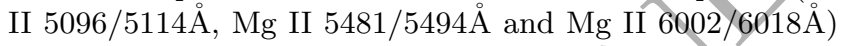
were unresolved by RSS. This, coupled with the higher noise of the RSS spectra, meant that the higher equiyalent width uncertainties precluded making any quantifiable conclusion on any line strength changes between the RSS and FORS2 observations.

\section{MEERKAT RADIO OBSERVATIONS}

GRB radio afterglows can probe the properties of the jet until very late times, when the jet essentially becomes nonrelativistic. The distribution of afterglow radio detection times, after trigger, for radio-detected GRBs peaks between 16 and $32 \mathrm{~d}$, and detections have been made hundreds of days after trigger in some cases (Chandra \& Frail 2012). The typieal peak flux density is $\sim 100 \mu \mathrm{Jy}$ at $8.5 \mathrm{GHz}$ and $\sim 10 \mathrm{~d}$ after trigger. The radio flux typically declines as $t^{-1}$ after the peak. The radio afterglow of GRB 191221B was detected by ATCA 0.73 days after the GRB, at 5.5, 9.0, 16.7, and 21.2 $\mathrm{GHz}$ (Laskar 2019). This therefore motivated the attempt to

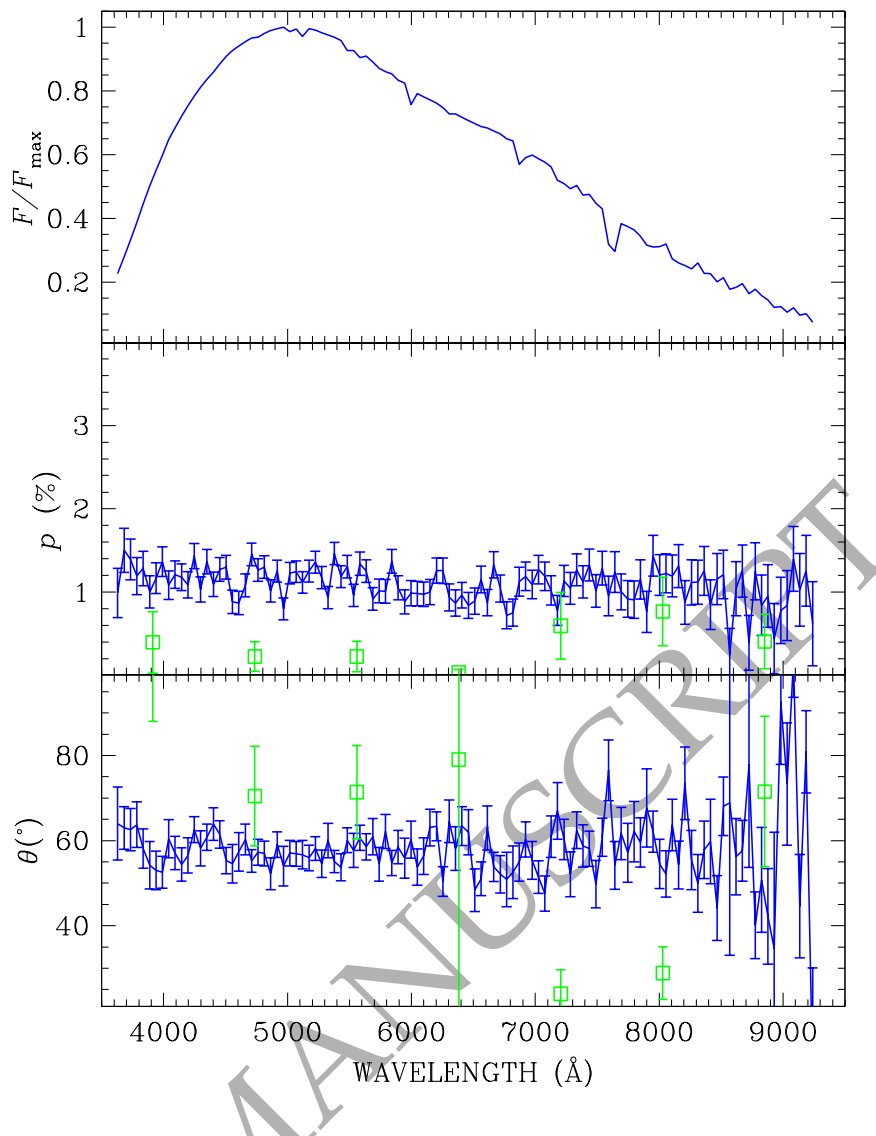

Figure 4. VLT/FORS2 spectropolarimetry of GRB 191221B covering 3600-9200 $\AA$ (blue symbols). Data have been rebinned at $50 \AA$. Green empty squares show the polarisation of a foreground star, rebinned at $825 \AA$.

observe GRB 191221B with the MeerKAT radio telescope array (Jonas 2009), in order to detect and monitor any radio emission from this GRB.

Observations of GRB 191221B with the MeerKAT radio telescope were attempted on 21 January 2020, from 20:26 to 21:26 UTC ( 30 d after the trigger) and 10 March 2021, from 17:33 to 18:32 UTC ( $\sim 44 \mathrm{~d}$ after the trigger), under Director's Discretionary Time (Monageng et al. 2020). We used J0408-6545 as the bandpass and flux calibrator, which was observed for $10 \mathrm{~min}$ at the start of the observations. The phase calibrator used was J1120 - 2508, which was observed for $2 \mathrm{~min}$ before and after the two $\sim 20 \mathrm{~min}$ scans of GRB 191221B in both observations (from 20:41:04.0-21:00:55.5 and 21:03:51.5-21:23:50.9 UTC on 21 January 2020 and 17:46:19.4-18:06:10.9 and 18:09:14.8-18:29:06.3 UTC on 10 March 2021, respectively). The observations were performed with 60 antennae and were centered at a frequency of $1.28 \mathrm{GHz}$ with a bandwidth of $856 \mathrm{MHz}$ over 4096 channels. The data were reduced using standard procedures in CASA (McMullin et al. 2007). The data were first flagged making use of AOFlagger (McMullin et al. 2007). Thereafter, phaseonly and antenna-based delays were corrected for making use of a model based on the primary calibrator. The bandpass correction for the relative system gain over the frequency range of the observation was determined and then complex gains were solved for the primary and secondary calibrators, 


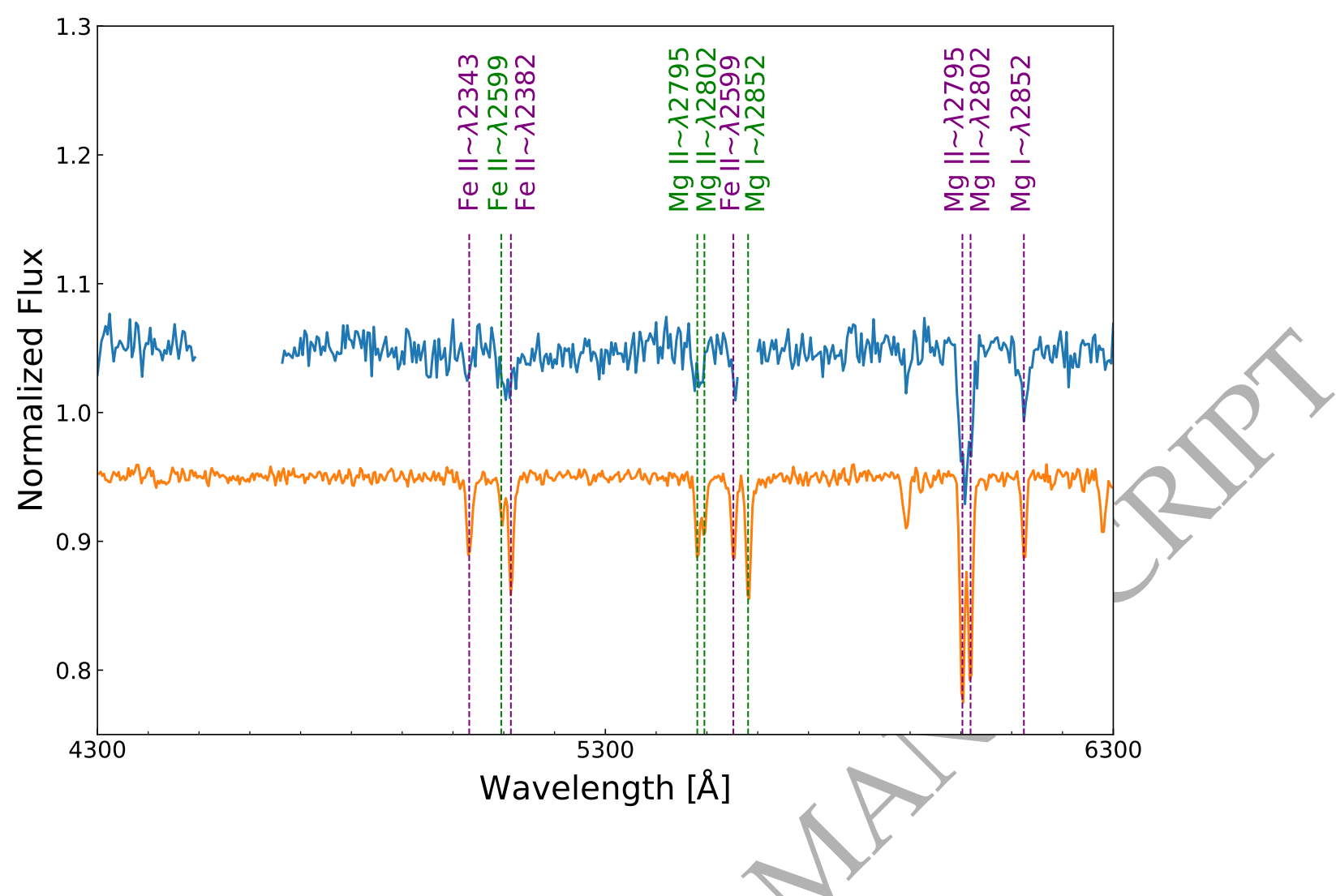

Figure 5. SALT/RSS (blue) and VLT/FORS2 (orange) spectra, normalised and offset by \pm 0.05 , respectively. Chip gaps and regions of sky subtraction are omitted from the RSS spectra. Absorption lines from both the host and an intervening galaxy are indicated. The unmarked line at $\approx 5900 \AA$ is Na D ISM absorption. Wavelength labels for the host galaxy lines are in purple while those for the intervening galaxy are in green.

Table 2. Measurements of spectral lines detected in the optical afterglow of GRB 191221B for the FORS2 observation. Lines corresponding to two redshifts are seen.

\begin{tabular}{cccccc} 
Line ID & $\begin{array}{c}\text { Rest Wavelength } \\
(\AA)\end{array}$ & $\begin{array}{c}\text { Observed Wavelength } \\
(\AA)\end{array}$ & $\begin{array}{c}\text { FWHM } \\
(\AA)\end{array}$ & $\begin{array}{c}\text { EW } \\
(\AA)\end{array}$ & z \\
\hline Fe II & 2343 & $5032.35 \pm 1.44$ & $5.44 \pm 1.43$ & $0.83 \pm 0.26$ & 1.148 \\
Fe II & 2599 & $5096.67 \pm 2.19$ & $4.70 \pm 2.26$ & $0.45 \pm 0.23$ & 0.961 \\
Fe II & 2382 & $5114.49 \pm 0.90$ & $4.67 \pm 0.96$ & $1.10 \pm 0.23$ & 1.147 \\
Mg II & 2795 & $5481.56 \pm 1.57$ & $4.10 \pm 1.67$ & $0.67 \pm 0.21$ & 0.961 \\
Mg II & 2802 & $5494.59 \pm 2.19$ & $3.93 \pm 2.29$ & $0.45 \pm 0.19$ & 0.961 \\
Fe II & 2599 & $5552.48 \pm 1.25$ & $4.01 \pm 1.25$ & $0.62 \pm 0.19$ & 1.136 \\
Mg I & 2852 & $5581.78 \pm 0.91$ & $4.44 \pm 0.91$ & $1.05 \pm 0.22$ & 0.957 \\
Mg II & 2795 & $6002.98 \pm 0.37$ & $4.56 \pm 0.40$ & $2.08 \pm 0.25$ & 1.148 \\
Mg II & 2802 & $6018.71 \pm 0.14$ & $4.38 \pm 0.43$ & $1.83 \pm 0.24$ & 1.148 \\
Mg I & 2852 & $6124.64 \pm 1.28$ & $4.10 \pm 1.30$ & $0.69 \pm 0.22$ & 1.128 \\
\hline
\end{tabular}

before scaling the gain corrections for the secondary calibrator from the primary calibrator and applying all the calibrations. Lastly, a small fraction of data were flagged using the RFLAG and TFCROP algorithms. Imaging was done using DDFacet (Tasse et al. 2018) and self-calibration using the
killMS software ${ }^{4}$ with the COHJONES solver. We choose ro-

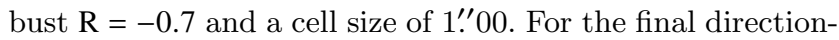
independent self-calibrated images, we estimate a rms noise of $\sim 17 \mu \mathrm{Jy} /$ beam and $\sim 13 \mu \mathrm{Jy} /$ beam for the observations performed on 21 January 2020 and 10 March 2021, respec-

${ }^{4}$ https://github.com/saopicc/killMS 
tively, within the vicinity of the source. The dimensions of the synthesised beam are $6.91^{\prime \prime} \times 4.44^{\prime \prime}$.

A source was detected at the nominal GRB 191221B afterglow position, with a peak flux density of $69 \pm 12$ $\mu \mathrm{Jy} /$ beam $(4.0 \sigma)$ and $47 \pm 11 \mu \mathrm{Jy} /$ beam $(\sim 3.6 \sigma)$ for the observations performed on 21 January 2020 and 10 March 2021, respectively. We show colour maps of the MeerKAT images of the GRB 191221B field in Figure 6, where the a radio source is clearly seen coincident with the optical position.

\section{MODELS}

The long GRB 191221B had a duration of $T_{90}=13.0 \pm 1.6 \mathrm{~s}$ (Sugita et al. 2019) with multiple pulses during the prompt phase. It was a very bright burst, with a $20 \mathrm{keV}-10 \mathrm{MeV}$ fluence of $(1.0 \pm 0.1) \times 10^{-4} \mathrm{erg} / \mathrm{cm}^{2}$ (Frederiks et al. 2019). Given the burst was located at a redshift of $z=1.148$ (Vielfaure et al. 2019), GRB 191221B was also rather energetic with $E_{\text {iso }}=(3.6 \pm 0.1) \times 10^{53}$ erg in the $1 \mathrm{keV}-10$ $\mathrm{MeV}$ rest-frame energy range (Frederiks et al. 2019).

Optical observations by MASTER show (see Figure 1) a declining flux $\left(F_{v} \propto t^{-\alpha}\right)$ from $t \gtrsim t_{0}+100 \mathrm{~s}$, typical of GRB afterglow emission (it is also likely there is an optical flare superposed on the decaying emission, but data coverage is sparse and no deeper conclusions can be drawn). The optical flux decay indices $\alpha_{\mathrm{OPT}}=-1.23 \pm 0.04$ until $t \sim t_{0}+3.5 \mathrm{ks}$ and $\alpha_{\mathrm{OPT}}=-0.88 \pm 0.02$ thereafter, until $t \sim t_{0}+10 \mathrm{ks}$ are compatible with synchrotron emission from a forward shock expanding into a constant-density interstellar medium (Sari 1999). In the slow-cooling regime $\left(v_{m}<v<v_{c}\right)$, the forwardshock model predicts $\alpha=3(p-1) / 4=0.90-0.98$ for the typically-assumed spectral index $p=2.2-2.3$ for the emitting electrons.

The optical flux decay index is comparable to the Swift XRT flux $(0.3-10 \mathrm{keV})$ decay index $\alpha_{X}=1.03_{-0.08}^{+0.09}$ after $t=t_{0}+4.9 \mathrm{ks}$ (D'Avanzo et al. 2019). The X-ray spectral index $\left(F_{v} \propto v^{-\beta}\right) \beta_{X}=0.86_{-0.06}^{+0.07}$ for the same Swift XRT flux (D'Avanzo et al. 2019) is also compatible with the expected value of $\beta=(p-1) / 2=0.60-0.65$, for $p=2.2 \rightarrow 2.3$, from the forward-shock model. Therefore we conclude that both optical and X-ray afterglows of GRB 191221B come from the slow-cooling segment of the synchrotron spectrum. This rules out a reverse-shock origin of the optical afterglow, which predicts $\alpha=(3 p+1) / 4=1.90$ for typical values of $p$ (Zhang et al. 2003).

The relative flattening of the optical light curve after $\sim t_{0}+10 \mathrm{ks}$ and subsequent decline is expected from the refreshed-shock scenario, where a slower GRB shell ejected later catches up with the decelerating outflow (Panaitescu et al. 1998). The light curve after $70 \mathrm{ks}$, steeply decaying as $t^{-1.96}$ as seeen in Figure 1 , is most-likely due to a jet break, which happens when the jet-opening angle $\theta_{\text {jet }} \sim 1 / \Gamma(t)$, where $\Gamma(t)$ is the bulk Lorentz factor of the jet. A jet break would cause the light curve to decay as $t^{-p}$ after $t \sim t_{\text {jet }}$ (Sari et al. 1999). Assuming the optical flux after $70 \mathrm{ks}$ is post-jet break, the spectral index of the emitting electrons is $p=\alpha=-1.96 \pm 0.14$. This is slightly harder than the $2.2-2.3$ values typically inferred from GRB afterglow modeling, but is consistent with generic particle acceleration models within the uncertainties.
The $69 \pm 12 \mu \mathrm{Jy}$ radio flux detected by MeerKAT at 1.28 $\mathrm{GHz}$ and $\sim 30 \mathrm{~d}$ after trigger is comparable to the $1.4 \mathrm{GHz}$ flux density measured from other GRBs (Chandra \& Frail 2012). However, our late-time observation at $\sim 444$ days shows that this flux stems mostly or completely from the host galaxy. We have estimated what the expected radio flux from star formation in a host galaxy at $z=1.2$ or an intervening galaxy at $z=0.96$. Using equation 1 presented in Berger et al. (2003), which is based on the original expression for the observed flux as a function of star formation rate (SFR) derived by Yun \& Carilli (2002), we calculated the required SFR needed to produce the observed MeerKAT flux. This was $53 \mathrm{M}_{\odot} \mathrm{y}^{-1}$, comparable to the SFR presented in Stanway et al. (2014) from radio observations of GRB hosts and consistent with radio observations of star forming region (Murphy et al. 2011).

The flux difference between the two MeerKAT observations is $22 \pm 16 \mu \mathrm{Jy}$, which may indicate an additional contribution from the radio afterglow, but the difference is not statistically significant. We cannot elaborate on the nature of the radio light curve and derive the power-law decline rate due to scarcity of other radio data points reported for this GRB to date. The flux of the first radio observation by ATCA, obtained $17.5 \mathrm{~h}$ after the trigger (Laskar 2019), is still to be published.

Synchrotron emission is expected to be highly polarised, although in the context of GRB emission models the expected degree of polarisation is $\lesssim 2 \%$ for a late afterglow (Covino \& Götz 2016). The reason is that, within the $1 / \Gamma(t)$ observable cone there can be a number of magnetic patches, each with a random orientation, thus reducing the degree of polarisation while adding emission incoherently (Gruzinoy \& Waxman 1999). This is particularly true for the forward-shock emission, which we believe is the origin of the observed optical emission in GRB 191221B, where the magnetic field is generated from turbulence and the magnetic patches are rather small (Medvedev \& Loeb 1999; Mao \& Wang 2017). Exotic effects such as mixing of photons with axion-like particles can also contribute to polarisation (Mena et al. 2011). The observed level of a few percent linear optical polarisation degree is therefore compatible with this qualitative scenario. An interesting effect is related to the viewing geometry of the afterglow near the time of the jet-break. Around this time, the polarisation components over the area of equal arrival time (an annulus in the case of a homogeneous top-hat jet with a magnetic field that is unordered) no longer sum to zero, and a marked change on the polarisation angle and degree is expected, depending of the viewing angle, the jet opening angle, the jet structure and the order of the magnetic field in the radiating surface (Rossi et al. 2004). This has been detected in some afterglows (e.g. Wiersema et al. 2014) but is not detected in all cases where polarimetry covers times near $t_{\text {jet }}$. We see no similar signature in the case of GRB 191221B. Unfortunately, the sparse polarimetric monitoring and the contribution due to dust-induced polarisation in the host galaxy prevents us to draw stronger conclusions. In fact, the various possible configurations model parameters can generate different scenarios for the polarisation time evolution, often with essentially the same flux evolution (e.g. Rossi et al. 2004; Covino \& Götz 2016; 

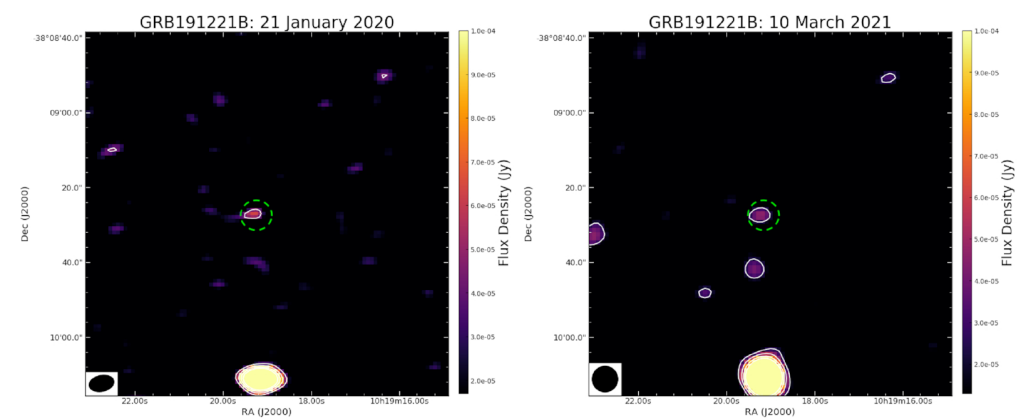

Figure 6. MeerKAT image $(1.5 \times 1.5)$ of the GRB 191221B field, centered at $1.28 \mathrm{GHz}$ and with a bandwidth of $0.86 \mathrm{GHz}$. The source near the center is coincident with the GRB 191221B position. The green dashed circle is centered at the most accurate position of GRB 191221B, determined from ALMA observations (Laskar \& a larger collaboration 2019). White contours are in multiples of $3 \sigma$ and the beam shape is shown in the bottom left corner.

Kobayashi 2019; Stringer \& Lazzati 2020; Shimoda \& Toma 2020; Teboul \& Shaviv 2020; Cheng et al. 2020).

In addition, if the surrounding medium is dense, we should further consider synchrotron polarisation radiative transfer in the optical band (Mao et al. 2018). The column density determined by the Swift-XRT observation provides an upper limit of $1.0 \times 10^{21} \mathrm{~cm}^{-2}$, and this corresponds to $A_{V}=0.56 \mathrm{mag}$. The relatively strong absorption leads to the low-degree polarisation of the observed continuum. Alternatively, the low-degree polarisation could be produced by the relativistic electron radiation in the stochastic magnetic field (Medvedev \& Loeb 1999; Sari 1999; Mao \& Wang 2017).

It is significant that some absorption lines are clearly detected in the observed spectra of GRB 191221B. Absorption by a patchy dense medium, permeated with a magnetic field, can be strongly amplified by relativistic shocks (Mizuno et al. 2014). When GRB shocks encounter a dense medium, we may consider the possibility of detecting polarisation features in the absorption lines. If the GRB jet is magnetically dominated, the bipolar magnetic field extending along the jet may reach the location of the absorbing material, although the strength of the magnetic field may decrease along the GRB jet. Some material may be ejected by the jet from the GRB central engine (Janiuk 2014; see also the recent work on baryon loading in relativistic magnetised shocks by Metzger et al. 2019). If particle cooling is effective, the optical photons can be absorbed by the cooled material. In the meanwhile, the magnetic field may have an effect on the material, even at a few parsecs from the GRB central engine.

The theoretical predictions mentioned above might be constrained by spectropolarimetric observations. Polarised radiative transfer of absorption lines was first mentioned by Unno (1956), where Zeeman splitting produces a triplet structure in a polarised absorption line. The detection of Zeeman split absorption lines is difficult and requires higher resolution spectral observations than in this study. We estimate that a spectral resolution of about $\mathrm{R} \sim 10^{5}$ will be necessary if we assume a magnetic field of about $10^{3} \mathrm{G}$ in the line-forming region (Mao et al. 2021). Although the detection of Zeeman splitting is a hard task, we believe that such a detection in GRB absorption lines in the optical band could be attempted in the future with sufficiently high-resolution spectropolarimetry on 8-m class telescopes, or larger.

\section{CONCLUSIONS}

We presented multi-epoch optical observations of the bright, long-duration GRB 191221B with MASTER, SALT \& VLT, as well as radio observations with MeerKAT. We obtained detailed photometric data with MASTER, while spectroplarimetric measurements were performed using data from SALT \& VLT. We detected emission at the position of GRB $191221 \mathrm{~B}$ with MeerKAT at $1.28 \mathrm{GHz}$ at a flux level of $\sim 70 \mu \mathrm{Jy}$ and $\sim 50 \mu \mathrm{Jy}$ at $30 \mathrm{~d}$ and $444 \mathrm{~d}$ post-burst, respectively, implying this to stem from the host galaxy of GRB 191221B, likely due to star formation.

The bright $(W=10.3 \mathrm{mag})$ prompt afterglow was detected with MASTER $38 \mathrm{~s}$ post-burst and monitored over $12 \mathrm{~h}$ to decline to $W=16.8 \mathrm{mag}$. The optical light curve after the prompt phase shows a smooth, power-law flux decay, as typically expected from GRB afterglow emission, with several breaks at later time. From the measured decline rates of the optical light curve and the close resemblance between the optical and Swift-XRT light curves, we conclude that the GRB 191221B optical afterglow is powered by slow-cooling synchrotron emission, ruling out a reverse-shock origin. The flattening and subsequent decline after $\sim 10 \mathrm{ks}$ is attributed to a refreshed-shock scenario, where a faster-moving shell ejected later catches up with the initial decelerated outflow. The steeper decay after $70 \mathrm{ks}$ is likely due to a jet break. We confirm that the inferred spectral index of radiating electrons is typical of the ones expected from the Fermi shockacceleration process.

Linear polarisation of optical emission from GRB 191221B was first detected by SALT/RSS at $\sim 1.5 \%$ some $\sim 3 \mathrm{~h}$ post burst, during a period when the brightness had plateaued. Observations with VLT/FORS2 showed little change in polarisation $\sim 10 \mathrm{~h}$ later, when GRB 191221B was on the decline. Such a low-level polarisation is expected for the late afterglow, when the emission is dominated by the forward shock with a randomly oriented magnetic field configuration.

GRB 191221B provided an opportunity to observe afterglow polarisation at late time. Our observations show that the degree of polarisation decreases marginally (by $\sim 0.3 \%$ ) over a timescale of $\sim 7 \mathrm{~h}$. Future spectro-polarimetric observations from early to late times could probe magnetic field structures in the reverse- and forward-shock regimes, and a transition from the former to the latter. 


\section{ACKNOWLEDGMENTS}

Some of the observations presented here were obtained with SALT under programme 2018-2-LSP-001 (PI: DAHB), which is supported by Poland under grant no. MNiSW DIR/WK/2016/07. Based on observations collected at the European Southern Observatory under ESO programme 0104.D-0600(C).

DAHB and JT acknowledge support through the National Research Foundation (NRF) of South Africa. MB is supported by the South African Research Chairs Initiative (grant no. 64789) of the Department of Science and Innovation and the NRF. ${ }^{5}$ DMH acknowledges financial support from the NRF and the SAAO. SR is partially supported by NRF with grant No. 111749 (CPRR) and by a University of Johannesburg Research Council grant. DAK acknowledges support from Spanish National Research Project RTI2018098104-J-I00 (GRBPhot). NPMK acknowledges support by the UK Space Agency. MASTER (equipment) is supported by Lomonosov Moscow State University Development Program. VL, DV are supported by RFBR grant 19-29-11011. CGM acknowledges financial support from Hiroko and Jim Sherwin.

We thank the Director and staff of SARAO for supporting our MeerKAT DDT observation. The MeerKAT telescope is operated by the South African Radio Astronomy Observatory (SARAO), which is a facility of the National Research Foundation, an agency of the Department of Science and Innovation.

\section{DATA AVAILABILITY}

The data underlying this article will be shared on reasonable request to the corresponding author. Some data (light curves and spectra) are available at the following: https://tinyurl.com/yyd3hty8

\section{REFERENCES}

Bagnulo S., et al., 2017, A\&A, 608, A146

Barth A. J., et al., 2003, ApJLett, 584, L47

Barthelmy S. D., et al., 2005, Space Sci. Rev, 120, 143

Berger E., Cowie L. L., Kulkarni S. R., Frail D. A., Aussel H., Barger A. J., 2003, ApJ, 588, 99

Bradley L., et al., 2016, Photutils: Photometry tools (ascl:1609.011)

Buckley D. A. H., Swart G. P., Meiring J. G., 2006, in Society of Photo-Optical Instrumentation Engineers (SPIE) Conference Series. p. $62670 Z$, doi: $10.1117 / 12.673750$

Burgh E. B., Nordsieck K. H., Kobulnicky H. A., Williams T. B., O'Donoghue D., Smith M. P., Percival J. W., 2003, in Iye M., Moorwood A. F. M, eds, Society of Photo-Optical Instrumentation Engineers (SPIE) Conference Series Vol. 4841, Instrument Design and Performance for Optical/Infrared Groundbased Telescopes. pp 1463-1471, doi:10.1117/12.460312

Burrows D. N., et al., 2005, Space Sci. Rev., 120, 165

Cano Z., Wang S.-Q., Dai Z.-G., Wu X.-F., 2017, Advances in Astronomy, 2017, 8929054

Any opinion, finding and conclusion or recommendation expressed in this material is that of the authors and the NRF does not accept any liability in this regard.
Chandra P., Frail D. A., 2012, ApJ, 746, 156

Cheng K. F., Zhao X. H., Bai J. M., 2020, MNRAS, 498, 3492

Covino S., Götz D., 2016, Astronomical and Astrophysical Transactions, 29, 205

D'Avanzo P., Laha S., Swift-XRT Team 2019, GRB Coordinates Network, 26559, 1

Evans P. A., et al., 2010, A\&A, 519, A102

Fossati L., Bagnulo S., Mason E., Land i Degl'Innocenti E., 2007, in Sterken C., ed., Astronomical Society of the Pacific Conference Series Vol. 364, The Future of Photometric, Spectrophotometric and Polarimetric Standardization. p. 503

Frederiks D., et al., 2019, GRB Coordinates Network, 26576, 1

Gaikwad R., Gupta S., Sharma V., Vibhute A., Bhattacharya D., Vadawale S., AstroSat CZTI Collaboration 2019, GRB Coordinates Network, 26567, 1

Gao H., Lei W.-H., Zou Y.-C., Wu X.-F., Zhang B. 2013, New Astron. Rev., 57, 141

Gehrels N., Razzaque S., 2013, Frontiers of Physics, 8, 661

Gehrels N., Ramirez-Ruiz E., Fox D. B., 2009, ARA\&A, 47, 567

Gendre B., 2019, GRB Coordinates Network, 26561, 1

Giannios D., Mimica P., Aloy M. A., 2008, A\&A, 478, 747

Gill R., Granot J., 2020, MNRAS, 491, 5815

Gomboc A., et al., 2008, ApJ, 687, 443

Gorbovskoy E., et al., 2010, Advances in Astronomy, 2010, 917584

Greiner J., et al., 2003, Nature, 426, 157

Gruzinov A., Waxman E., 1999, ApJ, 511, 852

Janiuk A., 2014, A\&A, 568, A105

Jonas J. L., 2009, IEEE Proceedings, 97, 1522

Jordana-Mitjans N., et al., 2020, ApJ, 892, 97

Kobayashi S., 2000, ApJ, 545, 807

Kobayashi S., 2019, Magnetic Fields in GammaRay Bursts and Their Polarised Emission. p. 337, doi:10.1007/978-3-030-19715-5_13

Kole M., et al., 2020, A\&A, 644, A124

Kong A. K. H., 2019, GRB Coordinates Network, 26566, 1

Kopač D., et al., 2015, ApJ, 813, 1

Kornilov W. G., et al., 2012, Experimental Astronomy, 33, 173

Kuin N. P. M., Swift/UVOT Team 2019, GRB Coordinates Network, 26538, 1

Kumar P., Zhang B., 2015, Phys. Rep., 561, 1

Laha S., Simpson K. K., Neil Gehrels Swift Observatory Team 2019, GRB Coordinates Network, 26534, 1

Laskar T., 2019, GRB Coordinates Network, 26580, 1

Laskar T., a larger collaboration 2019, GRB Coordinates Network, 26564, 1

Laskar T., et al., 2019, ApJLett, 878, L26

Lazzati D., et al., 2003, A\&A, 410, 823

Lipunov V., et al., 2010, Advances in Astronomy, 2010, 349171

Lipunov V. M., et al., 2019a, Astronomy Reports, 63, 293

Lipunov V., et al., 2019b, GRB Coordinates Network, 26536, 1

Lipunov V., et al., 2019c, GRB Coordinates Network, 26537, 1

Lipunov V., et al., 2019d, GRB Coordinates Network, 26555, 1

Longo F., et al., 2019, GRB Coordinates Network, 26549, 1

Lyutikov M., Pariev V. I., Blandford R. D., 2003, ApJ, 597, 998

Mao J., Wang J., 2017, ApJ, 838, 78

Mao J., Covino S., Wang J., 2018, ApJ, 860, 153

Mao J., Britto R., Buckley D. A. H., Covino S., D'Avanzo P., Kuin N. P. M., 2021, ApJ, in press

Maund J. R., Wheeler J. C., Patat F., Baade D., Wang L., Höflich P., 2007, A\&A, 475, L1

McMullin J. P., Waters B., Schiebel D., Young W., Golap K., 2007, in Shaw R. A., Hill F., Bell D. J., eds, Astronomical Society of the Pacific Conference Series Vol. 376, Astronomical Data Analysis Software and Systems XVI. p. 127

Medvedev M. V., Loeb A., 1999, ApJ, 526, 697

Mena O., Razzaque S., Villaescusa-Navarro F., 2011, J. Cosmology Astropart. Phys., 2011, 030 
Mészáros P., 2002, ARA\&A, 40, 137

Metzger B. D., Margalit B., Sironi L., 2019, MNRAS, 485, 4091

Mizuno Y., Pohl M., Niemiec J., Zhang B., Nishikawa K.-I., Hardee P. E., 2014, MNRAS, 439, 3490

Monageng I., Boettcher M., Buckley D. A. H., Britto R., Razzaque S., van Soelen B., 2020, GRB Coordinates Network, 26990, 1

Mundell C. G., et al., 2013, Nature, 504, 119

Murphy E. J., et al., 2011, ApJ, 737, 67

Nordsieck K. H., Jaehnig K. P., Burgh E. B., Kobulnicky H. A., Percival J. W., Smith M. P., 2003, in Fineschi S., ed., Society of Photo-Optical Instrumentation Engineers (SPIE) Conference Series Vol. 4843, Polarimetry in Astronomy. pp 170-179, doi:10.1117/12.459288

Panaitescu A., Mészáros P., Rees M. J., 1998, ApJ, 503, 314

Piran T., 1999, Phys. Rep., 314, 575

Piran T., 2004, Reviews of Modern Physics, 76, 1143

Rees M. J., Meszaros P., 1994, ApJLett, 430, L93

Romanov F. D., 2019, GRB Coordinates Network, 26565, 1

Roming P. W. A., et al., 2005, Space Sci. Rev., 120, 95

Rossi E. M., Lazzati D., Salmonson J. D., Ghisellini G., 2004, MNRAS, 354, 86

Sadovnichy V. A., et al., 2018, ApJ, 861, 48

Sakamoto T., Swift-BAT Team 2019, GRB Coordinates Network, 26562,1

Sari R., 1999, ApJLett, 524, L43

Sari R., Piran T., 1999, ApJ, 520, 641

Sari R., Piran T., Halpern J. P., 1999, ApJLett, 519, L17

Shimoda J., Toma K., 2020, arXiv e-prints, p. arXiv:2005.03710

Stanway E. R., Levan A. J., Davies L. J. M., 2014, MNRAS, 444, 2133

Steele I. A., Mundell C. G., Smith R. J., Kobayashi S., Guidorzi C., 2009, Nature, 462, 767

Stringer E., Lazzati D., 2020, ApJ, 892, 131

Sugita S., et al., 2019, GRB Coordinates Network, 26616, 1

Tasse C., et al., 2018, A\&A, 611, A87

Teboul O., Shaviv N., 2020, arXiv e-prints, p. arXiv:2008.10624

Toma K., Ioka K., Nakamura T., 2008, ApJLett, 673, L123

Troja E., et al., 2017, Nature, 547, 425

Unno W., 1956, PASJ, 8, 108

Urata Y., et al., 2019, ApJLett, 884, L58

Vielfaure J. B., et al., 2019, GRB Coordinates Network, 26553, 1

Wang L., Baade D., Hoeflich P., Wheeler J. C., 2003, arXiv eprints, pp astro-ph/0301266

Wang X.-G., et al., 2015, ApJS, 219, 9

Wiersema K., et al., 2012, MNRAS, 426, 2

Wiersema K., et al., 2014, Nature, 509, 201

Xue W. C., et al., 2019, GRB Coordinates Network, 26573, 1

Yun M. S., Carilli C. L., 2002, ApJ, 568,88

Zhang B., Kobayashi S., 2005, ApJ, 628, 315

Zhang B., Kobayashi S., Mészáros P., 2003, ApJ, 595, 950

van der Horst A. J., et al., 2014,MNRAS, 444, 3151 


\section{SUPPLEMENTARY MATERIAL}

Table 3 contains the light curve data used to produce Fig. 1

Table 3: Photometric data of GRB 191221B from MASTER Network

\begin{tabular}{|c|c|c|c|c|c|}
\hline JD & $\begin{array}{l}\text { Time }-\mathrm{T}_{0}^{*} \\
(\mathrm{sec})\end{array}$ & $\begin{array}{ll}\text { Exp } & \text { Time } \\
(\mathrm{sec}) & \\
\end{array}$ & Mag & Mag error & Telescope \\
\hline 2458839.360913 & 37.886 & 5 & 10.3 & 0.2 & $\begin{array}{l}\text { MASTER-SAAO } \\
\text { (VWFC) }\end{array}$ \\
\hline 2458839.361261 & 67.885 & 5 & 10.0 & 0.4 & $\begin{array}{l}\text { MASTER-SAAO } \\
\text { (VWFC) }\end{array}$ \\
\hline 2458839.361492 & 87.885 & 5 & 10.6 & 0.1 & $\begin{array}{l}\text { MASTER-SAAO } \\
\text { (VWFC) }\end{array}$ \\
\hline 2458839.361839 & 117.885 & 5 & 10.3 & 0.2 & $\begin{array}{l}\text { MASTER-SAAO } \\
\text { (VWFC) }\end{array}$ \\
\hline 2458839.362187 & 147.887 & 5 & 10.2 & 0.2 & $\begin{array}{l}\text { MASTER-SAAO } \\
\text { (VWFC) }\end{array}$ \\
\hline 2458839.362707 & 192.886 & 5 & 11.5 & 0.2 & $\begin{array}{l}\text { MASTER-SAAO } \\
\text { (VWFC) }\end{array}$ \\
\hline 2458839.363286 & 242.886 & 5 & 11.7 & 0.2 & MASTER-SAAO \\
\hline 2458839.381286 & 1798.113 & 180 & 14.70 & 0.03 & MASTER-SAAO \\
\hline 2458839.383617 & 1999.464 & 180 & 14.82 & 0.05 & MASTER-SAAO \\
\hline 2458839.385944 & 2200.491 & 180 & 14.96 & 0.04 & MASTER-SAAO \\
\hline 2458839.388273 & 2401.752 & 180 & 15.04 & 0.03 & MASTER-SAAO \\
\hline 2458839.390599 & 2602.750 & 180 & 15.18 & 0.02 & MASTER-SAAO \\
\hline 2458839.392927 & 2803.877 & 180 & 15.27 & 0.03 & MASTER-SAAO \\
\hline 2458839.395258 & 3005.248 & 180 & 15.31 & 0.04 & MASTER-SAAO \\
\hline 2458839.397602 & 3207.769 & 180 & 15.42 & 0.03 & MASTER-SAAO \\
\hline 2458839.399930 & 3408.877 & 180 & 15.48 & 0.03 & MASTER-SAAO \\
\hline 2458839.402257 & 3609.922 & 180 & 15.48 & 0.03 & MASTER-SAAO \\
\hline 2458839.404584 & 3811.038 & 180 & 15.54 & 0.03 & MASTER-SAAO \\
\hline 2458839.406912 & 4012.132 & 180 & 15.54 & 0.04 & MASTER-SAAO \\
\hline 2458839.409241 & 4213.369 & 180 & 15.60 & 0.03 & MASTER-SAAO \\
\hline 2458839.411570 & 4414.610 & 180 & 15.60 & 0.03 & MASTER-SAAO \\
\hline 2458839.413916 & 4617.299 & 180 & 15.67 & 0.03 & MASTER-SAAO \\
\hline 2458839.416244 & 4818.424 & 180 & 15.73 & 0.03 & MASTER-SAAO \\
\hline 2458839.418575 & 5019.806 & 180 & 15.83 & 0.03 & MASTER-SAAO \\
\hline 2458839.420901 & 5220.809 & 180 & 15.73 & 0.02 & MASTER-SAAO \\
\hline 2458839.423232 & 5422.215 & 180 & 15.82 & 0.03 & MASTER-SAAO \\
\hline 2458839.425559 & 5623.241 & 180 & 15.84 & 0.02 & MASTER-SAAO \\
\hline 2458839.427886 & 5824.315 & 180 & 15.86 & 0.03 & MASTER-SAAO \\
\hline 2458839.430216 & 6025.613 & 180 & 15.88 & 0.04 & MASTER-SAAO \\
\hline 2458839.432546 & 6226.953 & 180 & 15.94 & 0.02 & MASTER-SAAO \\
\hline 2458839.434874 & 6428.108 & 180 & 16.01 & 0.02 & MASTER-SAAO \\
\hline 2458839.437212 & 6630.034 & 180 & 16.01 & 0.03 & MASTER-SAAO \\
\hline 2458839.439543 & 6831.448 & 180 & 15.98 & 0.02 & MASTER-SAAO \\
\hline 2458839.441873 & 7032.811 & 180 & 16.06 & 0.03 & MASTER-SAAO \\
\hline 2458839.444201 & 7233.885 & 180 & 16.09 & 0.03 & MASTER-SAAO \\
\hline 2458839.446527 & 7434.918 & 180 & 16.01 & 0.03 & MASTER-SAAO \\
\hline 2458839.448858 & 7636.296 & 180 & 16.12 & 0.03 & MASTER-SAAO \\
\hline 2458839.451190 & 7837.810 & 180 & 16.12 & 0.03 & MASTER-SAAO \\
\hline 2458839.453547 & 8041.403 & 180 & 16.19 & 0.02 & MASTER-SAAO \\
\hline 2458839.455877 & 8242.746 & 180 & 16.26 & 0.03 & MASTER-SAAO \\
\hline 2458839.458206 & 8443.924 & 180 & 16.24 & 0.03 & MASTER-SAAO \\
\hline 2458839.460536 & 8645.301 & 180 & 16.24 & 0.02 & MASTER-SAAO \\
\hline 2458839.462864 & 8846.442 & 180 & 16.30 & 0.03 & MASTER-SAAO \\
\hline 2458839.465194 & 9047.694 & 180 & 16.30 & 0.03 & MASTER-SAAO \\
\hline 2458839.467524 & 9249.053 & 180 & 16.34 & 0.02 & MASTER-SAAO \\
\hline 2458839.469854 & 9450.312 & 180 & 16.35 & 0.03 & MASTER-SAAO \\
\hline 2458839.472181 & 9651.391 & 180 & 16.38 & 0.02 & MASTER-SAAO \\
\hline 2458839.474507 & 9852.379 & 180 & 16.36 & 0.03 & MASTER-SAAO \\
\hline
\end{tabular}


Buckley et al.

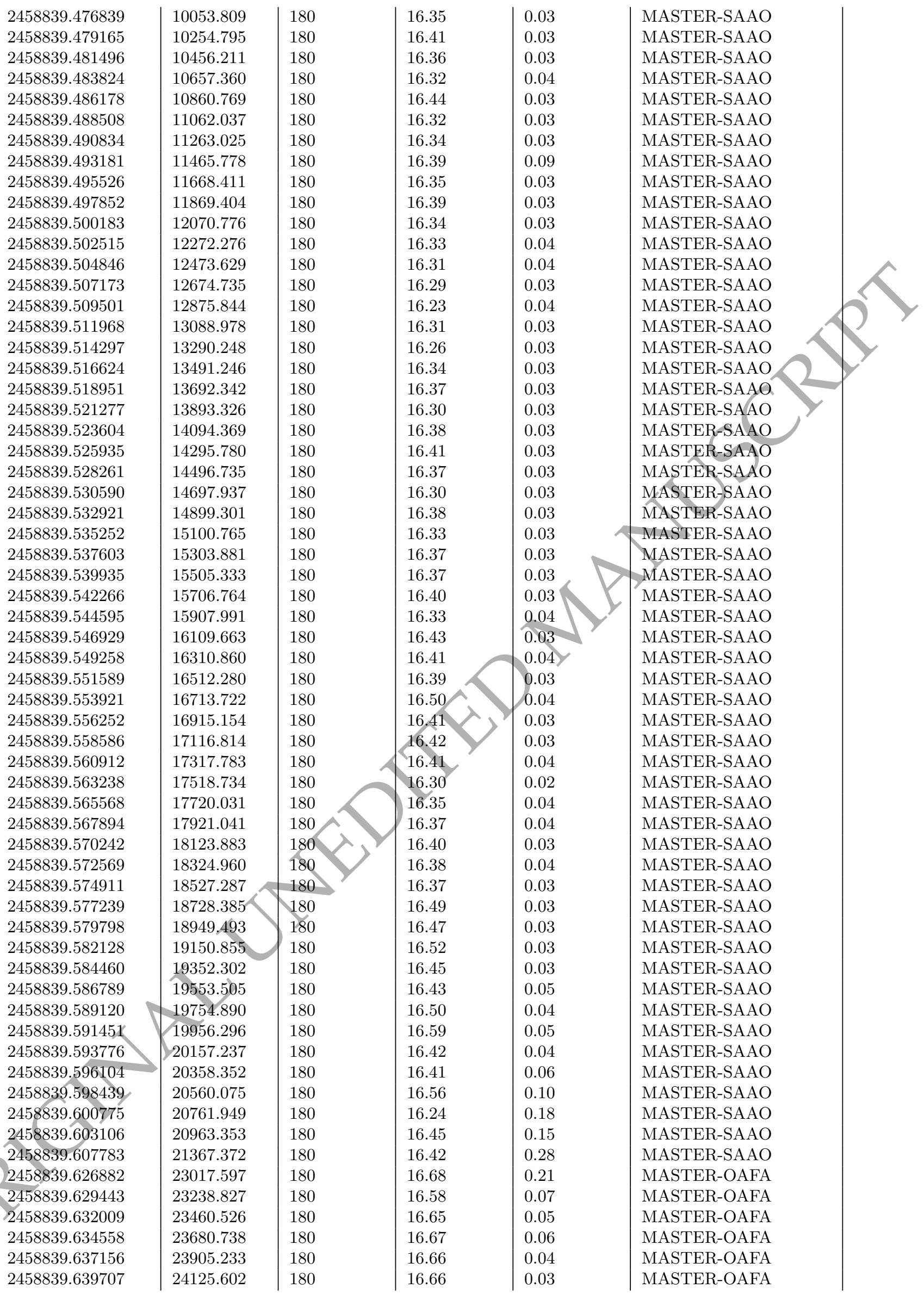




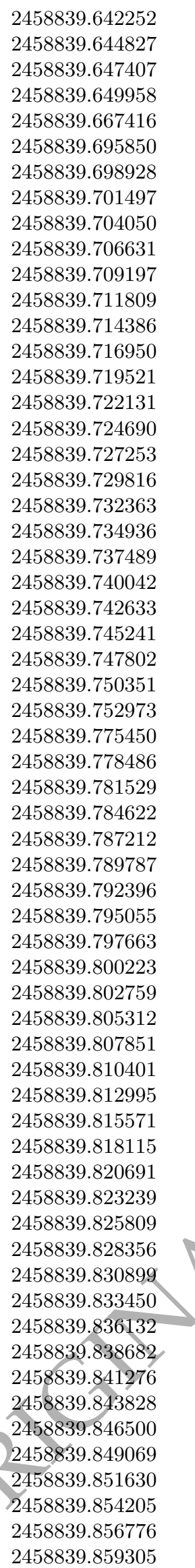

2458839.642252 2458839.644827 2458839.647407 2458839.698928 2458839.701497 (458839.704050 2458839.709197 2458839.711809 2458839.714386 2458839.716950 2458839.71952 2458839.724690 2458839.727253 2458839.729816 2458839.732363 2458839.734936 2458839.740042 2458839.742633 2458839.747802 2458839.750351 2458839.752973 2458839.775450 2458839.778486 2458839.784622 2458839.787212 2458839.789787 2458839.792396 2458839.795055 2458839.800223 2458839.802759 2458839.805312 2458839.807851 2458839.810401 2458839.815571 2458839.818115 2458839.823239 2458839.825809 2458839.830899 2458839.833450 2458839.838682 2458839.841276 2458839.843828 2458839.846500 2458839.849069 2458839.856776
2458839.859305

\begin{tabular}{|c|c|c|c|}
\hline 24345.503 & 180 & 16.60 & 0.04 \\
\hline 24568.042 & 180 & 16.59 & 0.03 \\
\hline 24790.890 & 180 & 16.70 & 0.04 \\
\hline 25011.358 & 180 & 16.63 & 0.05 \\
\hline 26519.669 & 180 & 16.59 & 0.06 \\
\hline 28976.410 & 180 & 16.75 & 0.10 \\
\hline 29242.351 & 180 & 16.74 & 0.04 \\
\hline 29464.291 & 180 & 16.77 & 0.04 \\
\hline 29684.852 & 180 & 16.78 & 0.03 \\
\hline 29907.875 & 180 & 16.62 & 0.03 \\
\hline 30129.602 & 180 & 16.65 & 0.03 \\
\hline 30355.230 & 180 & 16.62 & 0.03 \\
\hline 30577.933 & 180 & 16.67 & 0.02 \\
\hline 30799.477 & 180 & 16.68 & 0.03 \\
\hline 31021.601 & 180 & 16.65 & 0.02 \\
\hline 31247.108 & 180 & 16.71 & 0.02 \\
\hline 31468.190 & 180 & 16.68 & 0.03 \\
\hline 31689.622 & 180 & 16.66 & 0.03 \\
\hline 31911.019 & 180 & 16.69 & 0.02 \\
\hline 32131.087 & 180 & 16.72 & 0.03 \\
\hline 32353.405 & 180 & 16.69 & 0.02 \\
\hline 32574.006 & 180 & 16.71 & 0.03 \\
\hline 32794.579 & 180 & 16.69 & 0.03 \\
\hline 33018.483 & 180 & 16.73 & 0.03 \\
\hline 33243.775 & 180 & 16.72 & 0.03 \\
\hline 33465.080 & 180 & 16.76 & 0.05 \\
\hline 33685.274 & 180 & 16.60 & 0.11 \\
\hline 33911.863 & 180 & 16.79 & 0.08 \\
\hline 35853.819 & 180 & 16.95 & 0.13 \\
\hline 36116.156 & 180 & 16.92 & 0.20 \\
\hline 36379.022 & 180 & 16.92 & 0.05 \\
\hline 36646.263 & 180 & 16.80 & 0.11 \\
\hline 36870.063 & 180 & 16.88 & 0.02 \\
\hline 37092.529 & 180 & 16.88 & 0.10 \\
\hline 37318.001 & 180 & & 0.03 \\
\hline 37547.725 & 180 & 16.85 & 0.03 \\
\hline 37773.064 & 180 & & 0.02 \\
\hline 37994.188 & 180 & & 0.02 \\
\hline 38213.307 & 180 & 16.83 & 0.03 \\
\hline 38433.955 & 180 & 16.86 & 0.02 \\
\hline 38653.318 & 100 & 16.89 & 0.02 \\
\hline 38873.625 & 180 & 16.87 & 0.03 \\
\hline 39097.768 & 180 & 16.90 & 0.03 \\
\hline 39320.273 & 180 & 16.86 & 0.03 \\
\hline 39540,079 & 180 & 16.90 & 0.03 \\
\hline 39762.675 & 180 & 16.95 & 0.02 \\
\hline 39982.790 & 180 & 16.91 & 0.02 \\
\hline 40204.872 & 180 & 16.92 & 0.03 \\
\hline 40424.940 & 180 & 16.93 & 0.02 \\
\hline 40644.642 & 180 & 16.90 & 0.02 \\
\hline 40865.050 & 180 & 16.98 & 0.02 \\
\hline 41096.760 & 180 & 16.91 & 0.02 \\
\hline 41317.054 & 180 & 16.93 & 0.03 \\
\hline 41541.203 & 180 & 16.97 & 0.03 \\
\hline 41761.694 & 180 & 16.93 & 0.02 \\
\hline 41992.590 & 180 & 16.89 & 0.03 \\
\hline 42214.559 & 180 & 16.86 & 0.03 \\
\hline 42435.797 & 180 & 16.92 & 0.03 \\
\hline 42658.243 & 180 & 16.88 & 0.05 \\
\hline 42880.399 & 180 & 17.07 & 0.05 \\
\hline 43098.882 & 180 & 16.59 & 0.13 \\
\hline
\end{tabular}

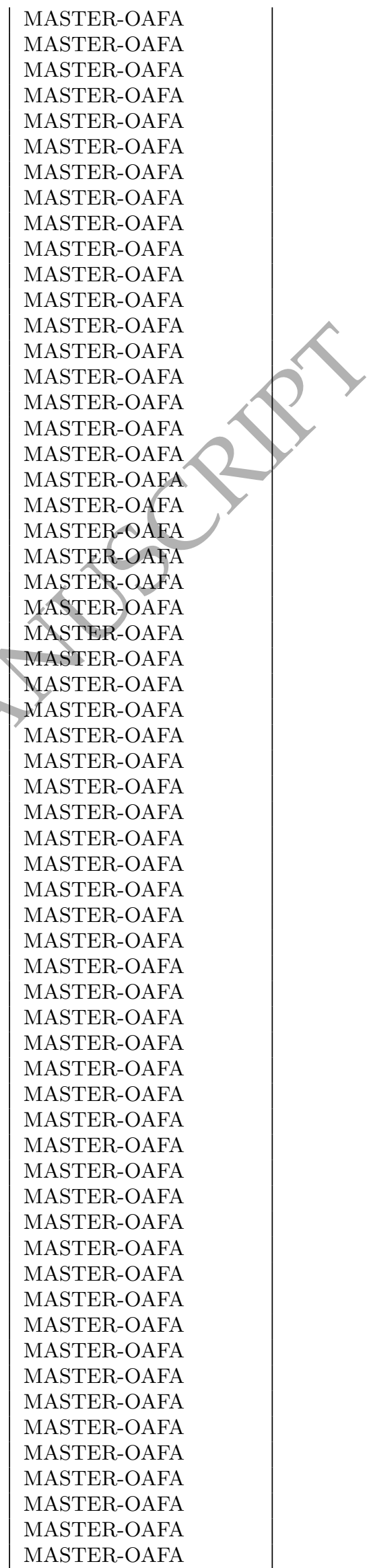

MASTER-OAFA MASTER-OAFA MASTER-OAFA MASTER-OAFA MASTER-OAFA MASTER-OAFA

MASTER-OAFA

MASTER-OAFA

MASTER-OAFA

MASTER-OAFA

MASTER-OAFA

MASTER-OAFA

MASTER-OAFA

MASTER-OAF

MASTER-OAFA

MASTER-OAFA

MASTER-OAFA

MASTER-OAFA

MASTER-OAFA

ER-OAFA

MASTER-OAFA

MASTER-OAFA

MASTER-OAFA

MASTER-OAFA

MASTER-OAFA

MASTER-OAFA

MASTER-OAFA

MASTER-OAFA

MASTER-OAFA

MASTER-OAFA

MASTER-OAFA

MASTER-OAFA

MASTER-OAFA

MASTER-OAFA

MASTER-OAFA

MASTER-OAFA

MASTER-OAFA

MASTER-OAFA

MASTER-OAFA

MASTER-OAFA 
16 Buckley et al.

${ }^{*}$ Burst time $=\mathrm{T}_{0}=$ JD 2458839.360475 (CALET, Sugita et al. 2019)

This paper has been typeset from a $\mathrm{T}_{\mathrm{E}} \mathrm{X} / \mathrm{LAT} \mathrm{EX}$ file prepared by the author. 